\title{
The complexity of homomorphisms of signed graphs and signed constraint satisfaction
}

\author{
Florent Foucaud ${ }^{1}$ and Reza Naserasr ${ }^{2}$ \\ 1 Universitat Politècnica de Catalunya, Barcelona (Spain) \\ University of Johannesburg, Auckland Park (South Africa) \\ PSL, Université Paris-Dauphine, LAMSADE - CNRS UMR 7243 (France) \\ florent. foucaud@gmail.com \\ 2 CNRS, Université Paris-Sud 11, LRI - CNRS UMR 8623, Orsay (France) \\ reza@lri.fr
}

\begin{abstract}
A signed graph $(G, \Sigma)$ is an undirected graph $G$ together with an assignment of signs (positive or negative) to all its edges, where $\Sigma$ denotes the set of negative edges. Two signatures are said to be equivalent if one can be obtained from the other by a sequence of resignings (i.e. switching the sign of all edges incident to a given vertex). Extending the notion of usual graph homomorphisms, homomorphisms of signed graphs were introduced, and have lead to some extensions and strengthenings in the theory of graph colorings and homomorphisms. We study the complexity of deciding whether a given signed graph admits a homomorphism to a fixed target signed graph $[H, \Sigma]$, i.e. the $(H, \Sigma)$-Coloring problem. We prove a dichotomy result for the class of all $\left(C_{k}, \Sigma\right)$-COLORING problems (where $C_{k}$ is a cycle of length $k \geq 3$ ): $\left(C_{k}, \Sigma\right)$-Coloring is NP-complete, unless both $k$ and the size of $\Sigma$ are even. We conjecture that this dichotomy can be extended to all signed graphs in a natural way. We also introduce the more general concept of signed constraint satisfaction problems and show that a dichotomy for such problems is equivalent to the statement of the Feder-Vardi Dichotomy Conjecture.
\end{abstract}

\section{Introduction}

The Four Color Theorem (4CT), stating that every planar graph is 4-colorable, is considered to be one of the central theorems in graph theory and, considering its simple statement in the form of a map coloring theorem, attracts a wide audience. One can reason the hidden beauty of this theorem in scientific ways based on the following classic theorems:

Theorem 1. Deciding if a given graph is 4-colorable is NP-complete.

Theorem 2. Deciding if a given planar graph is 3-colorable is NP-complete.

The latter indicates that the class of planar graphs (though recognizable in linear time) is a rich class of graphs, but the $4 \mathrm{CT}$ shows that 4-colorability for this rich class of graphs can be easily decided (simply answer YES all the time). This is in contrast with the former theorem. 
Despite being such a powerful theorem, the $4 \mathrm{CT}$ witnesses a special weakness. While it is very easily decidable if a graph is 2-colorable (i.e., bipartite), the 4CT proves no bound on the chromatic number of such a graph. A more complicated case is when an edge of a planar graph is replaced with a large (complete) bipartite graph. Such an operation does not change the chromatic number of the graph, but makes it far from being planar. Attempts to strengthen the $4 \mathrm{CT}$ so that it provides some bound in such cases has developed the theory of signed graphs. Coloring of graphs with signed graphs as forbidden minors have been studied, see for example Odd Hadwiger's conjecture (we refer to [8] for some recent developments), an extension of the well-known Hadwiger conjecture. Only recently, the development of the theory of homomorphisms of signed graphs has begun, see [1014]. This paper is the first study of the complexity of signed graph homomorphisms. This work is also strongly related to the celebrated Dichotomy Conjecture of Feder and Vardi 7]. We proceed with some notation.

Given a graph $G$, a signature is an assignment of signs + and - to the edges of $G$. It is normally denoted by the set $\Sigma$ of negative edges (the others being positive). Given a graph together with a signature, a resigning at a vertex $v$ is to change the sign of all edges incident to $v$. Two signatures $\Sigma_{1}$ and $\Sigma_{2}$ are said to be equivalent if one can be obtained from the other by a sequence of resignings - equivalently, by changing the signs at the edges of an edge-cut of $G$. This is an equivalence relation on the class of all signatures of a graph. A signed graph is defined to be a graph together with a class of equivalent signatures. It will normally be denoted by $[G, \Sigma]$ where $\Sigma$ is any member of the equivalence class of signatures. When we want to emphasize on a specific signature, say $\Sigma_{1}$, then we will write $\left(G, \Sigma_{1}\right)$. Note that one can easily check in polynomial time whether two signatures are equivalent using a reduction to 2-SAT or using Theorem 4

Proposition 3. Let $G$ be a graph, and let $\Sigma$ and $\Sigma^{\prime}$ be two signatures of $S$. One can decide in polynomial time whether $\Sigma \equiv \Sigma^{\prime}$.

An important notion here is the one of balance of a cycle. A cycle with even number of negative edges is called balanced cycle and the ones with odd number of negative edges are unbalanced cycles. The set of balanced or unbalanced cycles of a signed graph uniquely determines the equivalent class of signatures by the following theorem of Zaslavsky.

Theorem 4 (Zaslavsky [15]). Given two signatures $\Sigma$ and $\Sigma^{\prime}$ on a graphs we have $\Sigma \equiv \Sigma^{\prime}$ if and only if the set of balanced (unbalanced) cycles are the same.

A minor of signed graph $(G, \Sigma)$ is a signed graph $\left(H, \Sigma^{\prime}\right)$ which is obtained from $(G, \Sigma)$ by a sequence of the following operations: $i$. deleting vertices or edges, $i i$. contracting a positive edge (that is to identify two end vertices and delete loops) and iii. resigning. The last operation implies that notion of minor for $(G, \Sigma)$ is the same as that of $[G, \Sigma]$. Using this notion, a strengthening of the $4 \mathrm{CT}$ (which corresponds to one of the first cases of Odd Hadwiger's conjecture) was announced by Guenin in 2005 [9]: If $(G, E(G))$ has no $\left(K_{5}, E\left(K_{5}\right)\right)$-minor, 
then $G$ is 4-colorable. Moreover, it follows from a recent work [6] that deciding if $(G, E(G))$ has a $\left(K_{5}, E\left(K_{5}\right)\right)$-minor is polynomial-time solvable.

A classic way of extending the theory of graph colorings is through graph homomorphisms. The extension to signed graphs, introduced in [10] is given below. Given two signed graphs $[G, \Sigma]$ and $\left[H, \Sigma_{1}\right]$ we say there is a homomorphism of $[G, \Sigma]$ to $\left[H, \Sigma_{1}\right]$, and write $[G, \Sigma] \rightarrow\left[H, \Sigma_{1}\right]$, if there is a mapping $\phi$ of $V(G)$ to $V(H)$ such that $i$. $\phi$ preserves the adjacency (i.e., $x y \in E(G) \Rightarrow \phi(x) \phi(y) \in$ $E(H)$, and $i i$. with respect to some choice of signature $\Sigma^{\prime} \equiv \Sigma, \phi$ also preserves the signs. Since the existence of a homomorphism does not depend on the signature of the target graph, we may write it as homomorphism to $\left(H, \Sigma_{1}\right)$.

By considering signed graphs where all edges are of the same sign, we observe that graph homomorphisms are a special case of signed graph homomorphisms. From a complexity point of view, the following is then the first natural question to ask in the theory of signed graph homomorphisms.

$\left(H, \Sigma_{1}\right)$-COLORING

INSTANCE: A signed graph $[G, \Sigma]$.

QUESTION: Does $[G, \Sigma] \rightarrow\left(H, \Sigma_{1}\right)$ ?

The celebrated dichotomy result by Hell and Nešetřil [11] states that for any (non-signed) graph $H, H$-Coloring is polynomial-time if $H$ is bipartite (in which case it becomes equivalent to checking 2-colorability), and NP-complete otherwise. As an extension of this result, we believe that there is a also dichotomy in the signed case, i.e., that for any given $\left(H, \Sigma_{1}\right)$, either $\left(H, \Sigma_{1}\right)$-Coloring is polynomial-time solvable, or it is NP-complete. In fact, we believe that the problem is NP-complete unless $H$ is bipartite and $\left(H, \Sigma_{1}\right)$ has no unbalanced cycle (in which case $\Sigma_{1} \equiv \emptyset$ and thus the problem becomes again equivalent to checking 2-colorability). As we mentioned earlier, when $\Sigma \equiv \emptyset$ or when $\Sigma \equiv E(G)$, the problem is reduced to simple graph homomorphisms and the dichotomy holds.

We point out that the ability of resigning gives the signed homomorphism problem a different flavor than classical homomorphism problems. When we do not allow resigning, we would get the concept of two-edge-colored graph homomorphisms [2], whose complexity was studied in [3]. Already for two-edgecolored cycles, a complexity classification is difficult to obtain, but the problem is significantly different; for example, the case of a 4-cycle with three blue edges and one red edge is polynomial-time solvable, see [5. This is in contrast with the signed graph case, as we will see in Section 2

Our results and structure of the paper. We begin by proving a dichotomy for the set of $\left(C_{k}, \Sigma\right)$-Coloring problems in Section 2 where $C_{k}$ denotes a cycle on $k$ vertices. In Section 3 , we discuss the case where the target is a signed bipartite graph. Indeed it is known that this case already captures all usual graph homomorphism problems, making it a good candidate for an interesting subclass to study. We also give a few more examples of signed graphs for which the corresponding homomorphism problem is NP-complete. In Section 4 , we define a natural extension of signed graph homomorphisms to signed 
relational structures. We then prove that a complexity dichotomy for the class of signed constraint satisfaction problems exists if and only if Feder-Vardi's celebrated dichotomy conjecture holds. The paper is concluded in Section 5 .

\section{$2 \quad$ Mapping to signed cycles}

In this section, we determine the complexity of $(H, \Sigma)$-CoLORING when $H$ is a fixed cycle on $k$ vertices, $C_{k}$. Observe that there are only two signed graphs based on a cycle $C_{k}$ : a balanced cycle, denoted $B C_{k}$ and which is equivalent to $\left(C_{k}, \emptyset\right)$, and the unbalanced cycle, denoted $U C_{k}$, which is equivalent to $\left(C_{k},\{e\}\right)$ where $e$ is any edge of $C_{k}$. Furthermore, for odd values of $k$, the unbalanced cycle $U C_{k}$ is also equivalent to $\left(C_{k}, E\left(C_{k}\right)\right)$. Thus for odd values of $k$, mapping signed graphs to $B C_{k}$ or $U C_{k}$ is equivalent to mapping graphs to the odd cycle $C_{k}$, hence by Hell-Nešetřil's theorem [11] it is an NP-complete problem. For $B C_{k}$ with even values of $k$, the problem is equivalent to 2-coloring of graphs, thus it is polynomial-time solvable. The case that remains to study is $U C_{k}$ with even values of $k$. In this section, we prove that this is an NP-hard problem (even if the underlying graph of the input signed graph is of maximum degree 6).

Theorem 5. $U C_{2 k}$-Coloring is NP-complete for any $k \geq 2$, even when restricted to signed (bipartite) graphs of maximum degree 6 .

To prove Theorem 5 , we give a reduction from Monotone Not-ALL-EQUAL$3 \mathrm{SAT}^{3}$ which is NP-complete [13:

Monotone Not-All-Equal-3SAT

INSTANCE: A set $\mathcal{C}$ of monotone size-3-clauses from a set $X$ of boolean variables. QUESTION: Is there a boolean assignment of the variables of $X$ such that each clause contains at least one false and one true variable?

The main idea of our proof is that it uses the resigning of specific vertices as indication for the truth assignment of the corresponding variables.

Proof. Without loss of generality, when mapping to $U C_{2 k}$, we assume $V\left(U C_{2 k}\right)=$ $\{1, \ldots, 2 k\}$ and $U C_{2 k}$ has only edge $\{12\}$ in its signature (see Figure 1 ).

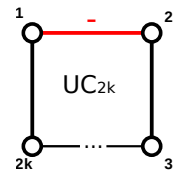

Fig. 1. The target graph $U C_{2 k}$.

Given a formula $F=\left\{C_{1}, \ldots, C_{m}\right\}$ over variable set $X$, we construct the signed graph $\left(G_{F}, \Sigma_{F}\right)$ as follows. For each clause $C=\left\{x_{1}, x_{2}, x_{3}\right\}$ of $F$ we

\footnotetext{
${ }^{3}$ Monotone: there are no negated variables.
} 
construct a clause gadget $\left(G_{C}, \Sigma_{C}\right): G_{C}$ has a central vertex, $c$; it contains three edge-disjoint copies $U_{1}, U_{2}, U_{3}$ of $U C_{2 k}$, meeting at vertex $c$ only. For each copy $U_{i}$, the unique vertex that is at distance $k$ of $c$ is denoted $x_{i}$, and corresponds to variable $x_{i}$ in clause $C$. For each $x_{i}$, we have a distinct path $P_{i}$ of length $k-1$ whose first end is identified with $x_{i}$, its other end being denoted $y_{i}$. Finally, let $U_{4}$ be a new cycle as follows: if $k=2, U_{4}$ is a copy of $U C_{6}$. Otherwise, $U_{4}$ is a copy of $U C_{2 k}$. We place $U_{4}$ such that it goes through $y_{1}, y_{2}$ and $y_{3}$ in such a way that the distances on $U_{4}$ between each pair of vertices $y_{i}, y_{j}$ are even, and two of them are equal to $\left\lfloor\frac{2 k}{6}\right\rfloor$ (i.e., the three distances are $\{2 \ell, 2 \ell, 2 \ell\}$ if $U_{4}$ has length $6 \ell,\{2 \ell, 2 \ell, 2 \ell+2\}$ if $U_{4}$ has length $6 \ell+2$, and $\{2 \ell, 2 \ell, 2 \ell+4\}$ if $U_{4}$ has length $6 \ell+4)$. Finally, we assign the following signature $\Sigma_{C}$ to $G_{C}: U_{1}, U_{2}, U_{3}$ contain exactly one negative edge each (this edge being incident to $c$ ); each path $P_{i}$ has exactly one negative edge (the one incident to $y_{i}$ ), and there is another negative edge incident to $y_{i}$ (the one of $U_{4}$ that lies on the path from $y_{i}$ to $y_{(i \bmod 3)+1}$ ). For $k=2$, the gadget is depicted in Figure 2 otherwise, see Figure 3(a).

Now, to build $\left(G_{F}, \Sigma_{F}\right)$, we consider all clause gadgets corresponding to distinct clauses and identify all vertices of type $c$ with each other. Vertices representing the same variable are identified with each other as well. $\Sigma_{F}$ is the union of all signatures $\Sigma_{C}$.

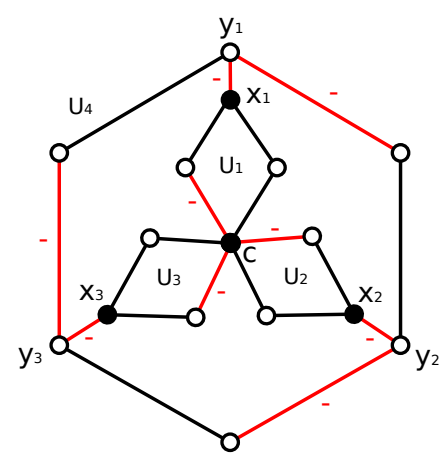

Fig. 2. Clause gadget for $U C_{4}$.

We now show that $\left(G_{F}, \Sigma_{F}\right)$ maps to $U C_{2 k}$ if and only $F$ is satisfiable. In the first part of the proof, no restriction on the maximum degree is shown; we explain afterward how to prove that part of the claim.

For the first part, assume that $F$ is satisfiable. We give a mapping $f$ from $\left(G_{F}, \Sigma_{F}\right)$ to $U C_{2 k}$ with the corresponding signature $\Sigma$ with $\Sigma_{F} \equiv \Sigma$.

Consider a truth assignment $A$ of $F$. We resign each vertex $x_{i}$ of $\left(G_{F}, \Sigma_{F}\right)$ such that the corresponding variable $x_{i}$ is true in $A$, and do not resign the vertices corresponding to a false variable. We also do not resign vertex $c$. Now, since $A$ is a satisfying truth assignment, either one or two variables are true in each clause. One can see that in any clause, it is possible to resign the remaining vertices as to obtain (up to symmetry) the signature with exactly four negative edges: one 


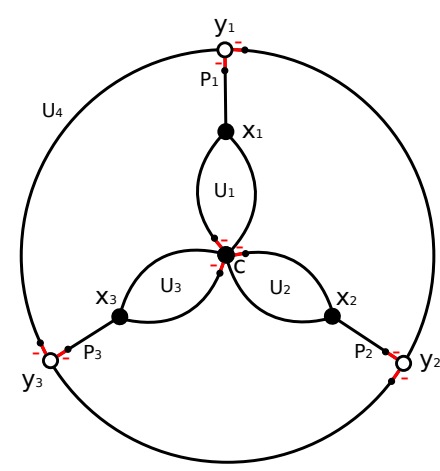

(a) Gadget for $U C_{2 k}, k \geq 3$.

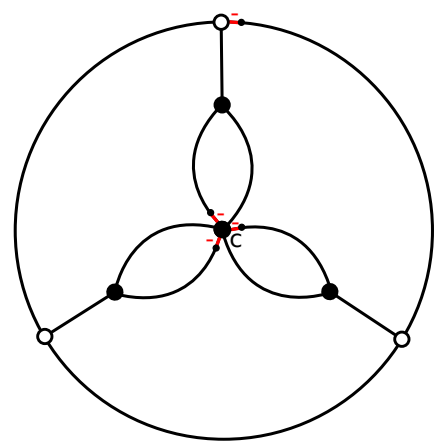

(b) Good signature for $G_{C}$.

Fig. 3. Clause gadget $G_{C}$ for larger cycles.

in each cycle $U_{i}(1 \leq i \leq 3)$ being incident to $c$, and one in $U_{4}$ incident to some $y_{j}(1 \leq j \leq 3)$, see Figure $3(\mathrm{~b})$, Let $\Sigma$ be the union of all these clause gadget signatures, and let us now construct the mapping $f$.

We map vertex $c$ to vertex 1 in $U C_{2 k}$, and each vertex $x_{i}$ is mapped to vertex $k+1$. Observe that in $\Sigma$ and in each clause gadget, exactly one vertex among $y_{1}, y_{2}, y_{3}$ has an incident negative edge. Without loss of generality, we assume it to be $y_{1}$, the other cases follow by symmetry.

If $k=2$, we map $y_{1}$ to vertex 2 , whereas $y_{2}, y_{3}$ are both mapped to vertex 4. It is now easy to extend the mapping.

If $k \geq 3$, we map $y_{1}$ to vertex 2 (the $k$ vertices $x_{1}, \ldots, y_{1}$ of path $P_{1}$ are mapped to $k+1, k, \ldots, 2)$. Let $\ell=\lfloor 2 k / 6\rfloor$. We now distinguish two cases.

On the one hand, if vertices $y_{1}, y_{2}$ and $y_{3}$ are pairwise at distance $2 \ell$ on the cycle $U_{4}$ (i.e. $U_{4}$ has length $2 k=6 \ell$ ), we map vertex $y_{2}$ to vertex $2 \ell+2$, and vertex $y_{3}$ to vertex $4 \ell+2$. Note that the parity of the length of each $P_{i}(k-1)$ is the same as the parity of the distance $d$ between vertices $k+1$ and $2 \ell+2$ or $4 \ell+2$ in $U C_{2 k}$, with $d<k$. It is now easy to complete the mapping.

On the other hand, if $y_{1}$ is at distance $2 \ell+2$ of say $y_{2}, 2 k=6 \ell+2$ and $y_{1}$ is at distance $2 \ell$ of $y_{3}$ (respectively, $2 k=6 \ell+4$ and $y_{1}$ is at distance $2 \ell+4$ of $y_{2}$ ), we map $y_{2}$ to vertex $2 \ell+4$ and $y_{3}$ to vertex $4 \ell+4$ (resp. $2 \ell+6$ and $4 \ell+6$ ). Again it is now easy to complete the mapping.

For the other part, suppose that $\left(G_{F}, \Sigma_{F}\right)$ maps to $U C_{2 k}$. Let $f$ be the mapping, and $\Sigma$ the signature that corresponds to $f$. Without loss of generality, we can assume that vertex $c$ of $G_{F}$ maps to vertex 1 of $U C_{2 k}$ (if not, since $C_{2 k}$ is vertex-transitive, it is easily seen that we could resign $\left(G_{F}, \Sigma\right)$ in an appropriate manner so that this would hold for some other signature).

We claim that when obtaining $\Sigma$ from $\Sigma_{F}$, for each clause $C=\left\{x_{1}, x_{2}, x_{3}\right\}$, either one or two of the vertices $x_{1}, x_{2}, x_{3}$ of $G_{C}$ have to be resigned. In this case, setting to TRUE each variable $x_{i}$ such that the corresponding vertex $x_{i}$ has been resigned, and to FALSE otherwise, would yield a truth assignment satisfying $F$. 
Observe first that the three cycles starting at vertex $c$ are unbalanced and of length $2 k$. Hence, they have to map to $U C_{2 k}$ in a surjective way, and each vertex $x_{i}$ maps to vertex $k+1$ of $U C_{2 k}$. Hence, the path joining vertex $x_{i}$ to vertex $y_{i}$ has to map to a path of $U C_{2 k}$ having only positive edges, because the distance between $x_{i}$ and $y_{i}$ is exactly $k-1$. Therefore, vertex $x_{i}$ is resigned if and only if vertex $y_{i}$ is not resigned. Indeed, if $y_{i}$ is not resigned, the edge incident to $y_{i}$ on the path from $y_{i}$ to $x_{i}$ remains negative. Since each edge of this path maps to a positive edge, all vertices of the path, including $x_{i}$, must be resigned. The other side follows from the same argument applied to the other end of the path.

We now claim that either one or two of the vertices $y_{1}, y_{2}, y_{3}$ of $G_{C}$ have to be resigned, which will complete the proof of this part.

If $k=2, y_{1}, y_{2}$ and $y_{3}$ lie on an unbalanced 6-cycle which hence has to map to $U C_{4}$ in a surjective way. The only way that this is possible is to map a path of length 3 of the 6 -cycle to an edge of $U C_{4}$. Assume, by contradiction, that all three vertices $y_{1}, y_{2}, y_{3}$ have been resigned. Then, no matter how the resigning is done on the other three vertices, it is not possible to proceed to the mapping of any such path of length 3 to an edge, since the three edges of this path should all have the same sign. The case where none of them is resigned follows by symmetry because the resulting signatures on the 6-cycle are symmetric.

Now, if $k \geq 3, U_{4}$ has to map in a surjective way to $U C_{2 k}$, and in the final signature $\Sigma$, exactly one of its edges must be negative. Assume that either none, or all three vertices $y_{1}, y_{2}, y_{3}$ have been resigned. Then the signature along $U_{4}$ does not change (up to symmetry): each of the paths $y_{1}, \ldots, y_{2}, y_{2}, \ldots, y_{3}$, $y_{1}, \ldots, y_{3}$ contains exactly one negative edge. But now, any resigning of the remaining vertices will lead to at least one negative edge on each of these three paths, a contradiction.

It now remains to prove how to restrict the maximum degree of our construction. Observe that in the above reduction, the reason for having a high maximum degree is that we identify all vertices of type $c$ and all vertices $x_{i}$ with each other. Instead of doing so, we can use a replicator gadget of length $\ell$, consisting of a sequence of $i$ unbalanced $2 k$-cycles $V_{1}, \ldots, V_{i}$, where each cycle $V_{i}$ has vertex set $\left\{v_{i}^{1}, \ldots, v_{i}^{2 k}\right\}$ and an edge between two consecutive vertices on this cyclic order. Each edge $\left\{v_{i}^{1}, v_{i}^{2}\right\}$ is negative. Moreover, for each $1 \leq i \leq \ell-1, V_{i}$ and $V_{i+1}$ share their edge $\{1,2 k\}$ when $i$ is odd, and the edge $\{k, k+1\}$ otherwise. An illustration is given in Figure 4

Now, observe that in order to map a replicator gadget of length $\ell$ to $U C_{2 k}$ with the signature of Figure 1 for each fixed $j(1 \leq j \leq 2 k)$, all vertices $v_{i}^{j}$, $1 \leq i \leq \ell$ have to be identified with each other. Moreover, it can be easily checked that either all vertices of the gadget have to be resigned, or none. Now, consider the construction of $G_{F}$ described in the first part of the proof. Let $x_{i} \in X$, and let $\ell_{i}$ be the number of occurrence of variable $x_{i}$ in $F$. Instead of identifying all vertices $x_{i}$ with each other, we take a copy $R_{i}$ of the replicator gadget of length $2 \ell_{i}$. Now, for the $j$ 'th clause $C$ containing $x_{i}$, we identify vertex $x_{i}$ of $G_{C}$ with vertex $v_{2 j-1}^{k+1}$ of $R_{i}$, as indicated in Figure 1. Moreover, we take an additional copy $R_{c}$ of the replicator gadget of length $6|F|$, where $|F|$ is the 


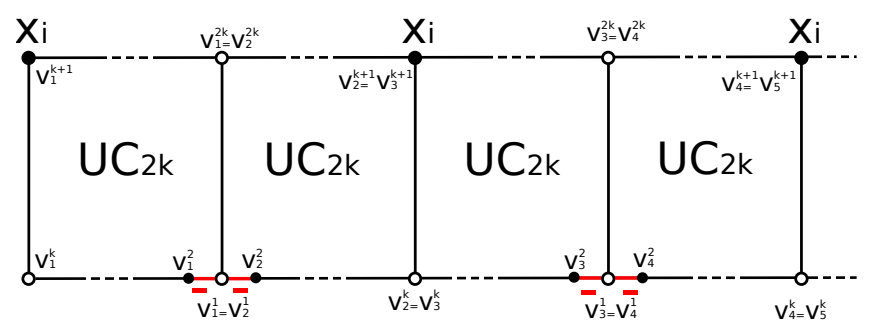

Fig. 4. Replicator gadget.

number of clauses in $F$. For each clause $C_{j}$, we split vertex $c$ of $G_{C_{j}}$ into three non-adjacent vertices $c_{1}, c_{2}, c_{3}$, each one being part of one of the cycles $U_{1}, U_{2}, U_{3}$, and we identify vertex $c_{i}(1 \leq i \leq 3)$ with vertex $v_{2(j+i-1)}^{1}$. Observe that the created graph has maximum degree 6 (vertices $x_{i}$ possibly having six neighbours: three in the clause gadget, and three in the replicator gadget).

By the properties of the replicator gadget, in a mapping from $G_{F}$ to $U C_{2 k}$, all vertices $c_{i}$ will be mapped to vertex 1 of $U C_{2 k}$, and vertices $x_{i}$, to vertex $k+1$, as in the original construction. Moreover, every vertex $x_{i}$ is resigned if and only if every other vertex $x_{i}$ is resigned, and the same holds for all vertices of type $c$. Hence the same proof as earlier applies.

\section{$3 \quad$ Further cases and signed bipartite graphs}

There are two special classes of signed graphs: signed graphs where, in some representation of signature, all edges are negative and signed bipartite graphs. These two are exactly the class of signed graphs in which all balanced cycles are even and all unbalanced cycles have a same parity. A homomorphism problem to a signed graph of former type is simply a graph homomorphism problem as all edges must be negative and then resigning does not play a role. In contrast, for the latter family, normally it is the choice of right signature that is the most difficult. However this case this case is already more difficult than graph homomorphism and graph coloring problems. It is shown in [14 that the concept of homomorphisms of signed bipartite graphs captures both the notion of homomorphisms of graphs and the concept of the chromatic number using the following construction. These theorems are stated based on the following construction of signed graphs from graphs: given a graph $G$, the signed bipartite graph $S(G)$ is obtained by replacing each edge $u v$ of $G$ by an unbalanced 4-cycle on four vertices $u x_{u v} v y_{u v}$, where $x_{u v}$ and $y_{u v}$ are new and distinct vertices. The following two theorems are then proved.

Theorem 6 (Naserasr, Rollová, Sopena [14]). For any graph $G, \chi(G) \leq k$ if and only if $S(G) \rightarrow\left(K_{k, k}, M\right)$, where $M$ is a perfect matching of $K_{k, k}$.

Theorem 7 (Naserasr, Rollová, Sopena [14]). For every pair $G, H$ of graphs, $G \rightarrow H$ if and only if $S(G) \rightarrow S(H)$. 
Note that if $[G, \Sigma] \rightarrow\left(H, \Sigma^{\prime}\right)$, then, in particular, $G \rightarrow H$. Therefore, if $H$ is a bipartite graph, this mapping would imply that $G$ is also a bipartite graph. Hence for bipartite signed graphs, the complexity of $([H, \Sigma)$-Coloring is determined by its complexity when reduced to signed bipartite input graphs. The above mentioned theorems then imply that $(H, \Sigma)$-COLORING is NP-complete whenever $(H, \Sigma)$ is $\left(K_{k, k}, M\right)$ for $k \geq 3$, or if $(H, \Sigma)$ is equivalent to $S(G)$ for any non-bipartite graph $G$.

Moreover, using Theorem 5, one can build more examples of signed graphs for which the homomorphism problem is NP-hard:

Theorem 8. $\left(K_{4},\{e\}\right)$-COLORING is NP-complete, where e is any edge of $K_{4}$.

Proof. Let $x, y, z, t$ be the four vertices of $K_{4}$ and assume $e=x y$. Let $U C_{4}$ be a signed cycle on $x, y, z$ and $t$ where $x y$ is a negative edge and $y z, z t, t x$ are positive edges (thus an unbalanced 4-cycle). We claim that a signed bipartite graph $[G, \Sigma]$ maps to $\left(K_{4},\{e\}\right)$ if and only if it maps to $U C_{4}$. Since $U C_{4}$ is a subgraph of $\left[K_{4},\{e\}\right]$, one direction is trivial. For the other direction, let $A, B$ be the bipartition of $G$ and let $\phi$ be the mapping of $[G, \Sigma]$ to $\left(K_{4},\{e\}\right)$ and suppose the mapping preserves the signs with respect to $\Sigma$. We define a new mapping $\phi^{\prime}$ which will be a homomorphism of $[G, \Sigma]$ to $U C_{4}$. For each vertex $u$ in $A$, if $\phi$ maps it to $x$ or $y$, then $\phi^{\prime}$ maps it to $x$, and if $\phi$ maps it to $z$ or $t$, then $\phi^{\prime}$ maps it to $z$. Similarly, for each vertex $v$ in $B$, if $\phi$ maps it to $x$ or $y$, then $\phi^{\prime}$ maps it to $y$, and if $\phi$ maps it to $z$ or $t$, then $\phi^{\prime}$ maps it to $t$. It can now be easily checked that $\phi^{\prime}$ is a homomorphism of $[G, \Sigma]$ to $U C_{4}$ with respect to $\Sigma$.

\section{Signed Constraint Satisfaction Problems}

A (finite) relational structure $T$ is a domain of elements, denoted $V(T)$, together with a finite set of relations $R_{1}, \ldots, R_{k}$, each relation $R_{i}(1 \leq i \leq k)$ having arity $a_{i}$ (that is, $R_{i} \subseteq V(T)^{a_{i}}$ ). An element of a relation $R_{i}$ is called a tuple. This is an extension of the notion of graphs and digraphs, as a graph is a relational structure with one binary and symmetric relation. When the symmetry is not forced we have the notion of digraphs.

The notion of homomorphisms of graphs and digraphs can then be generalized to relational structures as follows: given two relational structures $S$ and $T$ over the same number of relations $R_{1}, \ldots, R_{k}$ and the same (ordered) set of arities $a_{1}, \ldots, a_{k}$ a homomorphism of $S$ to $T$ is a mapping $\phi: V(S) \rightarrow V(T)$ such that if $X \in V(S)^{a_{i}}$ belongs to $R_{i}$ in $S$, then the ordered set $\phi(X)=$ $\{\phi(x), x \in X\}$ belongs to $R_{i}$ in $T$. We will write $S \rightarrow T$ whenever there exists a homomorphism of $S$ to $T$.

For every fixed relational structure $T$, we have the following associated decision problem, called T-CSP.

$T$-CSP

INSTANCE: A relational structure $S$.

QUESTION: Does $S$ admit a homomorphism to $T$ ? 
It is a folklore fact that this notion captures some well-known problems such as various versions of SAT-problems. The class of all constraint satisfaction problems is denoted by CSP. The complexity of CSPs has been extensively studied, see e.g. the book [1. One of the major open problems in the area is commonly known as the Dichotomy Conjecture, and has motivated many works such as e.g. [412]:

Conjecture 9 (Dichotomy Conjecture, Feder and Vardi [7]). For every fixed relational structure $T, T$-CSP is either polynomial or NP-complete.

Our aim in this section is to introduce the extended notion of signed relational structures and the related decision problem. We then show that a dichotomy for this class of problems is equivalent to the Dichotomy Conjecture.

A signed relational structure $(S, \Sigma)$ is a relational structure $S$ with a subset $\Sigma$ of the set of all tuples in $S$ (regardless of which relation the tuple belongs to). We say that the tuples in $\Sigma$ are negative, and the others are positive. Given an element $x$ in $S$, the resigning operation at $x$ switches the signs of all tuples in $S$ containing $x$. As for signed graphs, resigning defines an equivalence relation $\equiv$ between all signatures of $S$ : two signatures are equivalent if and only if they can be obtained from the other by a sequence of resignings. Relational structure with a class of equivalent signatures is then denoted by $[S, \Sigma]$.

We say that there is an homomorphism of $\left[S, \Sigma_{1}\right]$ to $[T, \Sigma]$ (or, equivalently, to $(T, \Sigma))$ if there is a signature $\Sigma_{1}^{\prime} \equiv \Sigma_{1}$ and a homomorphism $f: S \rightarrow T$ which preserves the signs of tuples according to $\Sigma_{1}^{\prime}$ and $\Sigma$.

Given a signed relational structure $(T, \Sigma)$, we define $(T, \Sigma)$-CSP analogously to $(H, \Sigma)$-COLORING:

$(T, \Sigma)-\mathrm{CSP}$

INSTANCE: A signed relational structure $\left[S, \Sigma_{1}\right]$.

QUESTION: Is there a homomorphism of $\left[S, \Sigma_{1}\right]$ to $(T, \Sigma)$ ?

We call the class of all signed constraint satisfaction problems $S$-CSP. We note that, by considering $\Sigma=\emptyset$ the class of signed constraint satisfaction problems contains the class of usual constraint satisfaction problems. Thus, a dichotomy for S-CSP would imply a dichotomy for CSP, i.e., the Dichotomy Conjecture. Our aim here is to show that the inverse is also true. This follows from another result of Feder and Vardi explained after the following definitions.

The class MMSNP, short for Monotone Monadic Strict NP, is the class of decision problems whose set of positive instances can be described in existential second-order logic with a universal first-order part (that is, having no existential quantifier). In other words, they can be described as the set of instances satisfying a formula of the form $F(S):=\exists S^{\prime}, \forall X, \Phi\left(X, S, S^{\prime}\right)$, where $S$ is the instance relational structure, $S^{\prime}$ is a relational structure $S^{\prime}$ with $V(S)=V\left(S^{\prime}\right)$ (intuitively, $S^{\prime}$ is the "proof" for $F(S)$ to be true), $X$ is a subset of elements in $V(S)$, and $\Phi$ is a first-order formula that only uses negations, disjunctions, conjunctions, relations of $S$ and $S^{\prime}$, and the equality operator. Moreover: 
- each relation of $S$ must appear in an odd number of negated subformulas in $\Phi$ (monotonicity);

- the relations in $S^{\prime}$ are only defined over sets of variables of $S$, not over relations of $S$ (monadicity);

- negation cannot be applied to the equality operator (no inequality).

It can be verified that each problem in CSP is also in MMSNP. Feder and Vardi, after introducing their Dichotomy Conjecture, proved that a dichotomy for CSP implies a dichotomy or MMSNP.

Theorem 10 (Feder and Vardi [7]). These three statements are equivalent: (i) MMSNP has a dichotomy;

(ii) CSP has a dichotomy;

(iii) the set of digraph homomorphism problems has a dichotomy.

Here we show that, furthermore, each S-CSP problem is also in MMSNP. Thus, while S-CSP includes CSP, it is included in MMSNP and, therefore:

Theorem 11. CSP has a dichotomy if and only if S-CSP has a dichotomy.

Proof. As we mentioned, it is enough to prove that each problem in S-CSP belongs to the class MMSNP.

First, consider a usual $T$-CSP problem, when there is no signature. For each instance $S$, the problem of deciding whether $S \rightarrow T$ can be express by a formula $F(S)$ in MMSNP: see 7 for details.

Now consider $(T, \Sigma)$-CSP and let $\left(S, \Sigma_{1}\right)$ be an input signed structure. To express $\left(S, \Sigma_{1}\right) \rightarrow(T, \Sigma)$ with a similar formula $F\left(S, \Sigma_{1}\right):=\exists S^{\prime}, \forall X, \Phi\left(X, S, S^{\prime}\right)$, one has to add that there is an assignment $s: V(S) \rightarrow\{0,1\}$ (which encodes the set of resigned elements, and can be expressed as a unary relation in $S^{\prime}$ ). Moreover, for each subset $X=\left\{x_{1}, \ldots, x_{k}\right\}$ of variables of $S$, not only $R_{i}(X)$ implies $R_{i}(f(X))$, but now also $(f(X), i) \in \Sigma$ implies that either $(X, i) \in \Sigma_{1}$ and an even number of elements in $X$ have been resigned, or $(X, i) \notin \Sigma_{1}$ and an odd number of elements in $X$ have been resigned. We give an example when $S, T$ have a unique binary relation $R$. The "proof" structure $S^{\prime}$ is a relational structure over $V(S)$ with a unary relation $A_{x}$ for every element $x$ in $V(T)$ (it encodes the assignment $V(S) \rightarrow V(T)$ ), and a unary relation $R_{S}$ (which encodes the set of resigned elements in $V(S))$.

$$
\begin{aligned}
& F\left(S, \Sigma_{1}\right):=\exists S^{\prime}, \forall\left(x_{1}, x_{2}\right) \in R, \\
& \left(\left(R\left(x_{1}, x_{2}\right) \wedge\left(x_{1}, x_{2}\right) \in \Sigma_{1} \wedge R_{S}\left(x_{1}\right) \wedge R_{S}\left(x_{2}\right)\right) \Rightarrow\left(\left(A_{x}\left(x_{1}\right) \wedge A_{y}\left(x_{2}\right)\right) \vee *\right)\right)
\end{aligned}
$$

[*: enumerate all assignations allowed by $T$ and belonging to $\Sigma$ ]

$$
\bigwedge \ldots \text { [repeat for each possibility for }\left(x_{1}, x_{2}\right) \in \Sigma_{1} \text { and resigning } x_{1} \text { and } x_{2} \text { ] }
$$

This formula is indeed monotone, monadic and without inequality. In fact, the only difference with a usual CSP is the case distinction according to which of $x_{1}, x_{2}$ have been resigned; hence the signature can simply be treated as additional constraints. 


\section{Conclusion}

As a first study of the complexity of signed graph homomorphisms, we have proved a dichotomy for signed cycles, by showing that $\left(C_{k}, \Sigma\right)$-COLORING is NPcomplete if and only if $\left(C_{k}, \Sigma\right)$ is both even and balanced. We have discussed some further cases, in particular, the case of signed bipartite graphs. As a natural generalization, the notion of signed constraint satisfaction problems and the corresponding class S-CSP were introduced, with S-CSP lying in between of the classes CSP and MMSNP. While it is a difficult problem to prove a dichotomy for S-CSP (equivalently, for CSP or for digraph homomorphism problems), it will be of interest to prove a dichotomy for signed graph homomorphism problems or other special cases of signed CSPs. By extending the classes in CSP/MMSNP for which a dichotomy is known, this would bring some new insight to the Dichotomy Conjecture, and therefore it is a promising direction of research.

\section{References}

1. Handbook of Constraint Programming (F. Rossi, P. van Beek, T. Walsh, eds.), Elsevier, 2006.

2. N. Alon and T. H. Marshall. Homomorphisms of edge-colored graphs and Coxeter groups. J. Algebr. Combin. 8(1)5-13, 1998.

3. R. C. Brewster and P. Hell. On homomorphisms to edge-coloured cycles. Electr. Notes Discrete Math. 5:46-49, 2000.

4. A. A. Bulatov. A dichotomy constraint on a three-element set. Proc. 43rd IEEE Symposium on Theory of Computing, 649-658, 2002.

5. C. Charpentier, R. Naserasr and E. Sopena. Analogue of Jeager-Zhang conjecture for signed bipartite graphs. Manuscript.

6. E. Demaine and M. Hajiaghayi and K-I. Kawarabayashi. Decomposition, Approximation, and Coloring of Odd-Minor-Free Graphs. Proc. SODA'10, 329-344, 2010.

7. T. Feder and M. Y. Vardi. The Computational structure of monotone monadic SNP and constraint catisfaction: a study through datalog and group theory. SIAM J. Comput. 28(1):57-104, 1998.

8. J. Geelen, B. Gerards, B. Reed, P. Seymour and A. Vetta. On the odd-minor variant of Hadwiger's conjecture. J. Combin. Theor. Series B 99:20-29, 2009.

9. B. Guenin. Graphs without odd-K5 minors are 4-colourable. Talk at Oberwolfach Seminar on Graph Theory, January 2005.

10. B. Guenin. Packing odd circuit covers: a conjecture. Unpublished manuscript, 2005.

11. P. Hell and J. Nešetřil. On the complexity of H-coloring. J. Combin. Theor. Series B 48(1):92-110, 1990.

12. P. Hell, J. Nešetřil and X. Zhu. Complexity of tree homomorphisms. Discrete Appl. Math. 70:23-36, 1996.

13. B. M. E. Moret. The Theory of Computation. Addison Wesley, 1998. Chapter 7, Problem 7.1, Part 2.

14. R. Naserasr, E. Rollová and E. Sopena. Homomorphisms of signed graphs. Submitted manuscript.

15. T. Zaslavsky. Signed graphs. Discrete Appl. Math. 4(1), 47-74, 1982. 